\title{
Influence of Shape and Orientation of Grinding Grains on Their Cutting Capacity
}

\author{
Dmitry Shatko ${ }^{1, *}$, Vladimir Lyukshin ${ }^{1}$, Pavel Strelnikov ${ }^{1}$, and Lyudmila Samorodova ${ }^{1}$ \\ ${ }^{1}$ T.F. Gorbachev Kuzbass State Technical University, Kemerovo 28, st. Vesennyaya, Kemerovo, \\ 650000, Russia
}

\begin{abstract}
The issues of the influence of orientation and the shape of abrasive grains on one of their most important operational characteristics cutting ability, and also on cutting properties of tools made of them, are considered in the article. Modern approaches to cutting capacity assessment, of both single grains, and loose abrasive mass are analyzed. The original method for the assessment of cutting capacity of a single grinding grain fixed in a rotating disk, implemented in surface grinding, is presented. The experimental data of the dependence of the cutting capacity on the shape and the orientation angle of the grinding grains are presented. In addition, the influence of the shape factor of the flap grinding wheel grains on its cutting capacity is shown. Practical recommendations on the application in practice of various varieties of grinding grains are proposed.
\end{abstract}

\section{Introduction}

The grinding process is characterized by a number of specific features that distinguish it from the cutting process with traditional blade tools, among which [1, 3, 6-8, 13-15]:

- behavior at relatively high cutting speeds;

- formation of chips of small length and cross section;

- cutting of a very thin layer of metal by grinding grain;

- simultaneous participation a large number of grinding grains in the cutting process;

- significant heating of the workpiece due to increased friction of the grinding tool on it;

- chaotic orientation of the grinding grains on the surface of the tool, as a result of which most of them do not participate in the cutting process, but subject the workpiece surface to elastic deformation.

A grinding wheel or belt is a multi-blade tool containing in its structure a large number of abrasive grains - cutting elements that are fixed together by a post. In the process of work, each single grain cuts its chip. Due to the fact that a large number of grains participate in the grinding process simultaneously, a significant removal of metal per unit time is ensured.

The above mentioned features of grinding lead to a number of difficulties in this behavior research. Proceeding from this, the cutting pattern is investigated mainly by

\footnotetext{
${ }^{*}$ Corresponding author: shdb.tm@kuzstu.ru
} 
indirect methods. The assessment methods for the cutting capacity of abrasives include [2, 9, 11]:

- abrasion of the disc with abradant;

- micro-cutting with a single grain, previously fixed in a rotating disk;

- micro-cutting with a single grain when implementing a linear motion scheme;

- wear of workpieces immersed in abrasive mass;

- abrasive machining of samples, implemented on a plant with grains fixed by centrifugal forces (pseudo-fixed layer);

- wear of samples placed in the gas flow of abrasive grains.

Since abrasive materials in one form or another are mainly used in grinding, it is logical to try to reproduce the actual grinding conditions when examining their cutting characteristics. Such conditions should include the following factors $[4,5,10,12]$ :

- the cutting speed is estimated at tens of meters per second;

- grinding is carried out mainly by fixed abrasive (the design of the tool provides for the presence of grain and post);

- there is some effort of pressing the working layer of grains to the material being machined;

- the process of micro-cutting during grinding is carried out by a large number of single grinding grains.

\section{Research methods}

The careful analysis of the approaches to assessing the cutting capacity in terms of the most accurate reproduction of real grinding conditions gives grounds to state that micro-cutting with a single grain, previously fixed in a rotating disc, is most preferable. Proceeding from this, this approach was taken as a basis for the assessment of cutting properties of single grains.

The experimental part of the work was carried out on a surface grinding machine model $3 \mathrm{G} 71$ by the means of the device designed for this purpose (Fig. 1).

The following cutting rates were selected experimentally:

- rotation speed of the disk with grinding grain fixed to it $30 \mathrm{~m} / \mathrm{s}$;

- longitudinal feed $0.19 \mathrm{~m} / \mathrm{min}$;

- number of passes - 20,

- depth of cut $0.04 \mathrm{~mm}$.

Flat (up) grinding was used as the cutting pattern.

When carrying out the experiments, the grinding grain 1 to be studied was fixed in the cage 2 by means of a post at a certain angle. After this, the cage was placed on the disk 3 , which, in turn, was fixed to the spindle. The sample to be worked 4 was weighed on a precise scale before the experiment, and then placed on the magnetic table of the grinding machine. The required feed amount and depth of cutting were set by the corresponding flywheels of the machine. The cutting depth was estimated by means of the dial gauge (DG). As the implementation of the 20 passes, the test sample was removed and reweighed. After which the experiment was repeated. Figure 2 shows the general view of the 3G71 machine with a tool mounted on the spindle. 


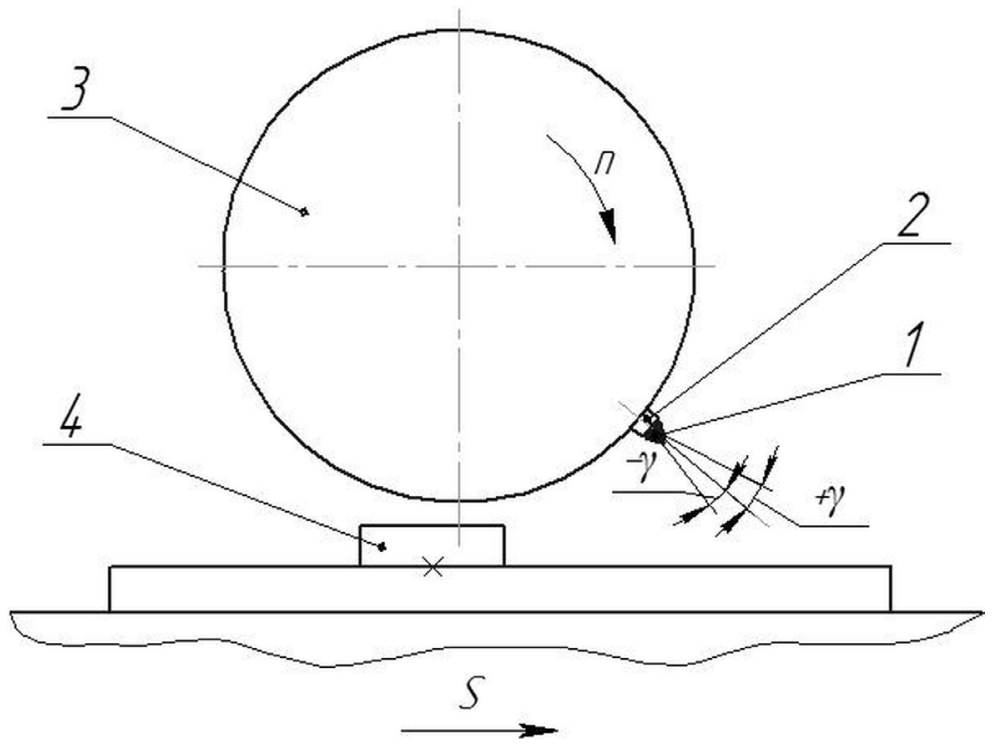

Fig. 1. Tool for assessing the cutting capacity of single grains.

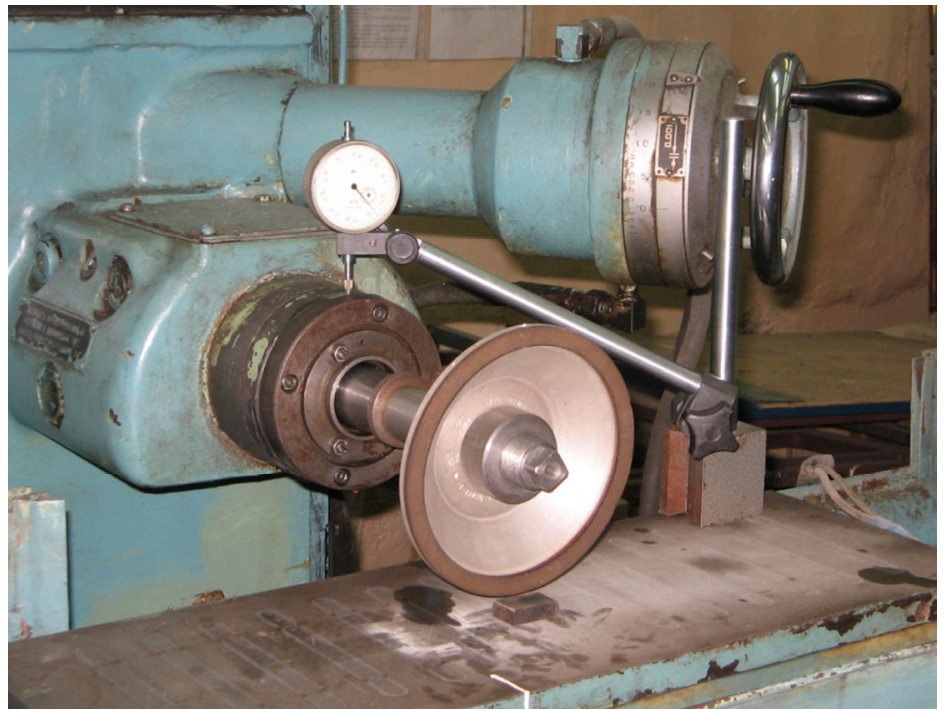

Fig. 2. General view of the machine $3 \mathrm{G} 71$ with the tool.

In the course of the experiments, 125 grit number 13A grade (regular alumina) abrasive grains of isometric $K_{f} \approx 1.14$ ), intermediate $K_{f} \approx 1.56$ ) and lamellar $K_{f} \approx 2.27$ ) shapes, manufactured by «Yurginsky abrasives», were used.

In order to manufacture the grinding grains of precisely specified shape, a specially designed separator was used for this purpose, with the aid of which a common abrasive mass was divided according to the shape of the mold into the required fractions. To determine the numerical values of the average shape factor of one or another fraction, obtained as a result of the screening, a specially developed computer program was used, which allows prompt calculating the numerical values of the shape factor of grinding grains. As for the orientation angle of the grinding grains $(\gamma)$, its changes from $0^{\circ}$ to $+90^{\circ}$ 
in the cutting direction were designed into the experiment, and it varied from $0^{\circ}$ to $-90^{\circ}$ in the opposite direction in $15^{\circ}$ increments.

With the preliminary sampling of materials for further research, the choice was made in favor of the material S235 of hardness HB 111. This is justified by the fact that experiments with other steels of higher hardness resulted in the destruction of the test single grinding grain. Metal bars with dimensions length $\mathrm{x}$ width $\mathrm{x}$ height $=30 \times 20 \times 10 \mathrm{~mm}$ were used as machined samples.

Before the experiment, each sample was subjected to pre-machining - grinding and finishing of the surface to be machined. The measurement of the mass of the removed material was carried out on the ADV-200 model scales, the weighing accuracy of which was $0.0005 \mathrm{~g}$.

After receiving an array of experimental data, the cutting capacity of grains was determined by the formula (1):

$$
\Delta M=M_{1}-M_{2},
$$

where $M_{1}$ and $M_{2}$ - sample weight before and after machining.

To ensure the statistical adequacy of the experimental data, each experiment was repeated at least 25 times. The research results were analyzed using the Microsoft Excel program.

\section{Result and discussion}

The processed experimental data are presented in the form of the graph shown in Fig. 3.

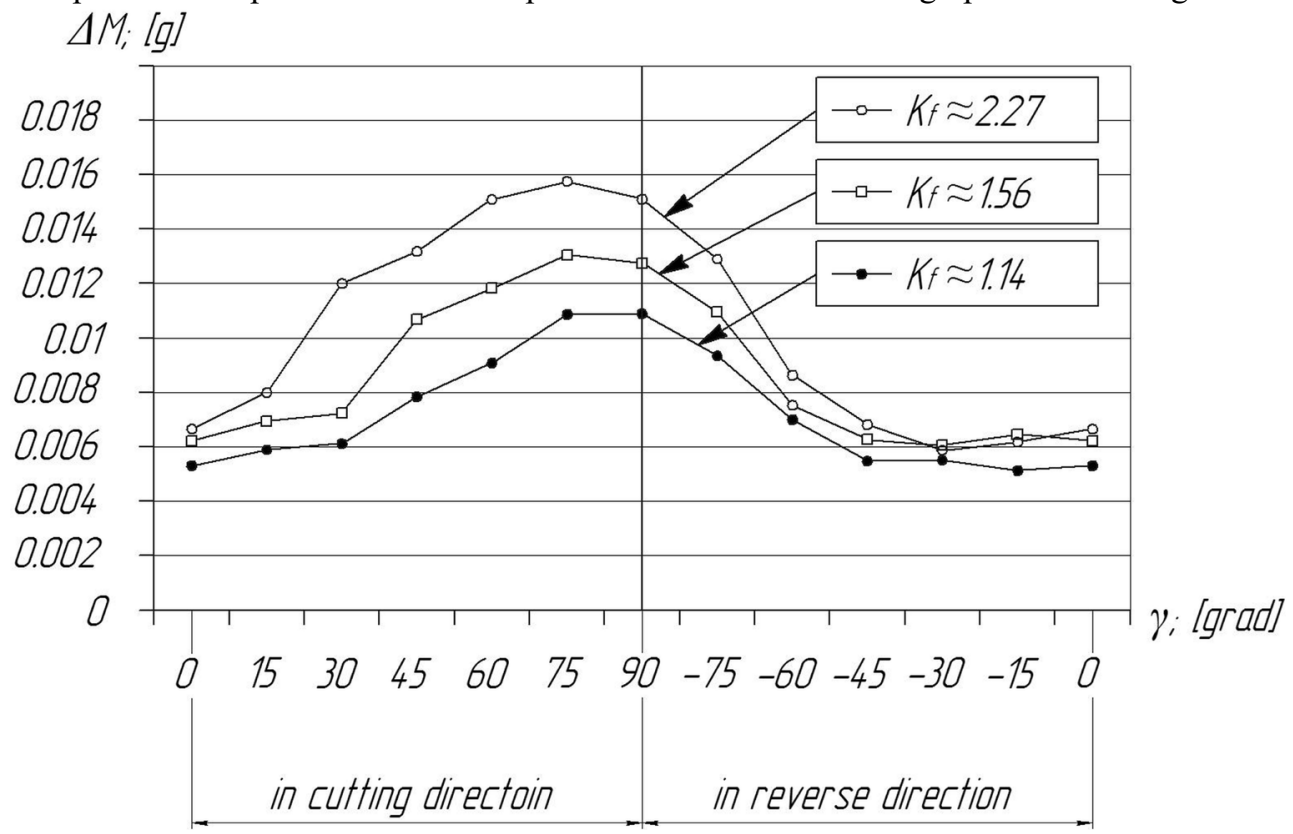

Fig. 3. Dependence of cutting capacity of abrasive grains on their shape and orientation angle.

The dependencies shown in the figure allow stating that:

- the orientation angle and the geometric shape of the grains affect their cutting capacity;

- abrasive grains of lamellar shape characterized by the greatest cutting capacity, and isometric grains - the smallest; 
- the greatest cutting capacity for different shapes of grains is provided by the following orientation angles: from $60^{\circ}$ to $90^{\circ}$ - for lamellar shape grains; from $60^{\circ}$ to $90^{\circ}-$ for intermediate shape grains; from $75^{\circ}$ to $90^{\circ}$ - for isometric shape grains.

The presented results make it possible to formulate a practical recommendation that, for increasing the cutting capacity of grinding tools, it is preferable to use acicular (lamellar) shape abrasive grains having an orientation angle of about $\gamma=75^{\circ}$.

To confirm the experimental data obtained in the case of single grinding grains, research aimed at studying the effect of the grinding grain shape on the cutting characteristics of grinding tools were also conducted. As an example, the dependence of the cutting capacity on the grinding grain shape when machining some grades of steel with flap wheel is shown in the figure:

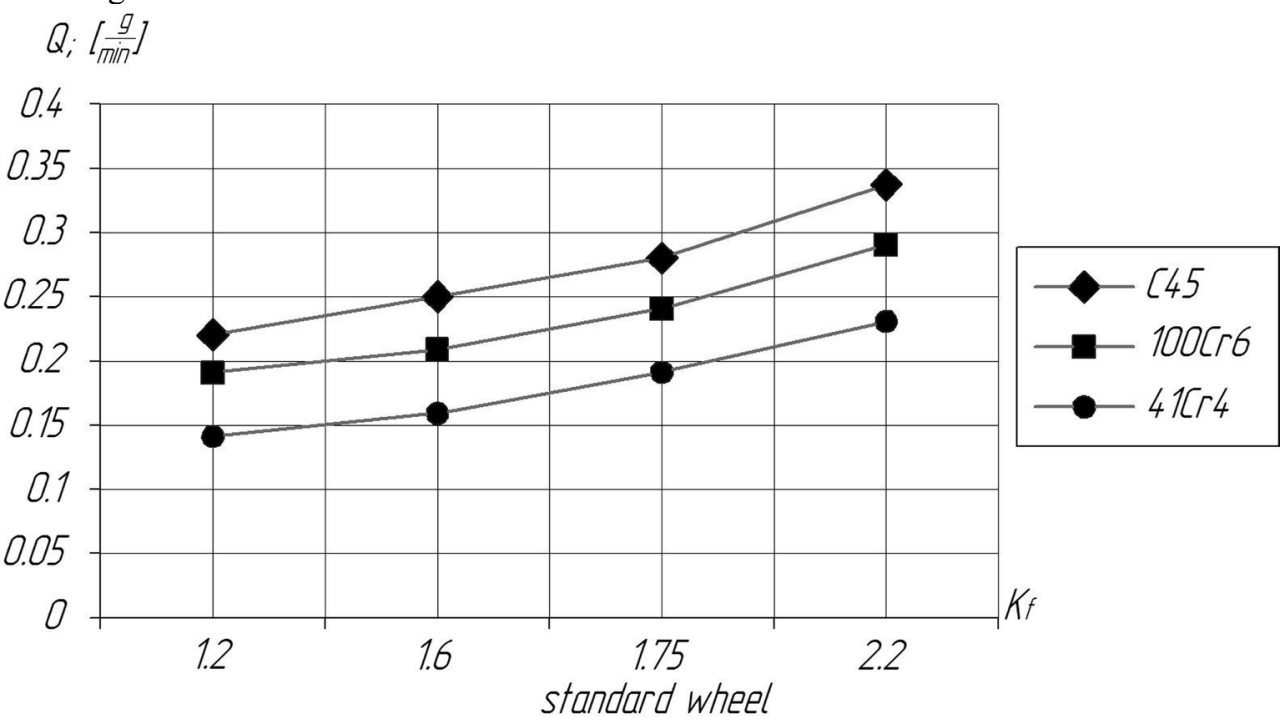

Fig. 4. Influence of the grinding grain shape on the cutting capacity of the flap wheels.

The presented dependences show that the transition from the isometric shape of the grains to the acicular (lamellar) leads to an increase in the cutting capacity curves for all grades of steel of the order of 1.56 times. When changing from a wheel made from a common abrasive mass with $K_{f} \approx 1.75$ to a wheel with $K_{f} \approx 2.2$, the cutting capacity increases by about 1.21 times. The transition in the opposite direction - to a wheel with $K_{f} \approx 1.2$ leads to a decrease in cutting capacity by an average of 1.29 times.

As for to the hardness of the workpiece being machined, its increase leads to a decrease in the cutting properties of the wheel. Thus, the transition from C45 (HB 187) in the delivery state to hardened 100Cr6 (HRC 65) is accompanied by a reduction in the cutting capacity of the order of 1.51 times.

The cutting capacity improvement in the transition from wheels made from isometric grains to wheels made from needle-shaped grains is explained in this way. Acicular (lamellar) grain has sharper corners, so during cutting it is better introduced into the material being machined. As a result, thicker chips are removed, compared to isometric grains, which have a larger front and lower back angles, which increases the level of plastic deformation, and also increases the friction of the wheel on the workpiece.

Thus, flap wheels containing isometric grains in their structure possess a lesser capacity to remove material in a unit of time compared to wheels made from acicular-shaped grains.

The dependence of the cutting capacity $(Q)$ on the grinding grain shape $\left(K_{f}\right)$ in the form of a mathematical model is presented below: 
or

$$
\operatorname{Ln}(Q)=0.79788 \cdot \ln \left(K_{f}\right)-1.70297
$$

$$
Q=\exp ^{(0.79788 \cdot \ln (K f)-1.70297)}
$$

An analysis of the obtained model shows that an increase in the value of the grain shape factor leads to an increase in the cutting capacity of the flap wheel.

\section{Conclusions}

The results of the research presented in this paper allow substantiating that the shape and orientation of grains that are used in the process of manufacturing grinding tools significantly affect their operational characteristics, in particular cutting capacity.

\section{References}

1. P. V. Arkhipov, A. S. Yanyushkin, D. V. Lobanov, S. I. Petrushin, AMM, 379 124130 (2013)

2. D. Shatko, V. Lyukshin, V. Bakumenko, IOP Conf. Series: Mater. Sci. Eng., 142 (2016)

3. A. Korotkov, L. Korotkova, R. Gubaidulina, AMM, 682, 469-473 (2014)

4. V. A. Korotkov, MTT, 1438150, 102-103 (2003)

5. Z. I. Kremen, D.A. Popovskii, V.G. Yur'ev, Russ. Eng. Res., 33, 457-459 (2013)

6. D. V. Lobanov, P. V. Arhipov, A. S. Yanyushkin, V. Y. Skeeba, IOP Conf. Series: Earth Environ. Sci., 87 (2017)

7. D. A. Rychkov, A. S. Yanyushkin, IOP Conf. Series: Mater. Sci. Eng., 142 (2016)

8. V. Korotkov, E. Minkin, IOP Conf. Series: Mater. Sci. Eng., 91, (2015)

9. V. Korotkov, S. Petrushin, AMM, 682, 224-230 (2014)

10. V. Lyukshin, AMM, 682, 148-153 (2014)

11. A. Kvasova, B. Gerike, E. Murko, D. SkudarnovE3S Web of Conferences 21, 03016 (2017)

12. A. S. Yanyushkin, D. A. Rychkov, Procedia Eng., 206, 944-949 (2017)

13. A. S. Yanyushkin, D. A. Rychkov, D.V. Lobanov, AMM, 682, 183-187 (2014)

14. P. V. Arkhipov, D. V. Lobanov, D. A. Rychkov, A. S. Yanyushkin, IOP Conf. Series: Mater. Sci. Eng., 327(4), 042009 (2018)

15. D. A. Rychkov, A. S. Yanyushkin, D. V. Lobanov, P. V. Arkhipov, IOP Conf. Series: Mater. Sci. Eng., 327(2), 022089 (2018) 